\title{
A Feasibility Study for Microwave Breast Cancer Detection Using Contrast-Agent-Loaded Bacterial Microbots
}

\author{
Yifan Chen, ${ }^{1}$ Panagiotis Kosmas, ${ }^{2}$ and Sylvain Martel $^{3}$ \\ ${ }^{1}$ Department of Electrical and Electronic Engineering, South University of Science and Technology of China, Shenzhen 518055, China \\ ${ }^{2}$ School of Natural and Mathematical Sciences, King's College London, London WC2R 2LS, UK \\ ${ }^{3}$ Department of Computer Engineering, Ecole Polytechnique de Montreal, Montreal, Canada H3T 1J4
}

Correspondence should be addressed to Yifan Chen; chen.yf@sustc.edu.cn

Received 25 June 2013; Accepted 24 September 2013

Academic Editor: Soon Yim Tan

Copyright (C) 2013 Yifan Chen et al. This is an open access article distributed under the Creative Commons Attribution License, which permits unrestricted use, distribution, and reproduction in any medium, provided the original work is properly cited.

\begin{abstract}
We propose a new approach to microwave breast tumor sensing and diagnosis based on the use of biocompatible flagellated magnetotactic bacteria (MTB) adapted to operate in human microvasculature. It has been verified experimentally by Martel et al. that externally generated magnetic gradients could be applied to guide the MTB along preplanned routes inside the human body, and a nanoload could be attached to these bacterial microbots. Motivated by these useful properties, we suggest loading a nanoscale microwave contrast agent such as carbon nanotubes (CNTs) or ferroelectric nanoparticles (FNPs) onto the MTB in order to modify the dielectric properties of tissues near the agent-loaded bacteria. Subsequently, we propose a novel differential microwave imaging (DMI) technique to track simultaneously multiple swarms of MTB microbots injected into the breast. We also present innovative strategies to detect and localize a breast tissue malignancy and estimate its size via this DMI-trackable bacterial microrobotic system. Finally, we use an anatomically realistic numerical breast phantom as a platform to demonstrate the feasibility of this tumor diagnostic method.
\end{abstract}

\section{Introduction}

In recent years, there have been considerable efforts in applying microwave technology for early-stage breast cancer detection [1]. This imaging modality attempts to discriminate breast tissues based on their dielectric properties, which are sensitive to physiological or pathological conditions such as water content, temperature, and vascularization. However, the effectiveness of microwave medical imaging is considerably influenced by clutter interference due to healthy tissue inhomogeneities [2]. This problem can be possibly solved by using a contrast agent such as carbon nanotubes (CNTs) [3] or ferroelectric nanoparticles (FNPs) [4] to change the tumor tissue dielectric values. These agents can be transported selectively to cancer cells via systemic administration for specific and noninvasive diagnosis of tissue malignancies within specified regions-of-interest (ROI). These contrast media can also be employed in medical therapeutic applications such as microwave hyperthermia treatment [3].
However, contrast-enhanced microwave cancer theranostics is compromised by currently available targeting techniques, which have limited efficacy due to intratumoral penetration limitations and the presence of quiescent cells within tumors [5]. As a result, only a small amount of contrast agent is able to enter cancer cells. Recent advance in micronano biorobotics has promised a potential remedy for this problem through the prospect of utilizing biocompatible flagellated magnetotactic bacteria (MTB) with nanometersized magnetosomes acting like computer-controlled medical microbots. Their swimming directions and displacement speeds can be regulated by an externally applied magnetic field $[6,7]$ to deliver effectively a nanoload such as contrast agents to a tumoral region in the human body, offering new possibilities for breast cancer detection in microwave tomographic [8] and radar-based [9] approaches. A tactic for MTB-oriented microwave breast cancer detection was suggested in [10], where each time a single swarm of MTB microbots loaded with a nanoscale contrast agent is injected 
into the breast from a predefined injection site. The microbots will navigate towards the direction of an externally exerted magnetic gradient and its movement could potentially be tracked by using the differential microwave imaging (DMI) algorithm proposed in [11]. In theory, when a particular microbot trajectory would meet a tumor, the contrast agent will be discharged from the microbots and attached to cancer cell receptors. Subsequently, these miniature biorobots will no longer be trackable by the DMI system, which only detects difference in the tissue dielectric properties caused by the agent. Therefore, a DMI footprint "sink" inside breast would emerge at the tumor location where the contrast agent accumulates eventually.

The current work extends the results in [10] by considering multiple agglomerations of MTB, which are injected concurrently into breast at each round of operation to reduce the diagnostic time. Our previous DMI algorithm [11] is only applicable to the location estimation of the nearest microbot swarm. Consequently, in order to track multiple swarms, the antennas of the DMI apparatus should be divided into several groups. Each group only tracks its nearest swarm. By enforcing the constraint that DMI footprints should be nearly aligned with the a priori known magnetic field, the average dielectric properties of breast can be approximated and the microbot swarms are tracked. Furthermore, as a significant extension to [12], we provide a comprehensive discussion on the main characteristics of the MTB as an ideal contrast-agent transporter and propose to deploy them to target possible tumor locations from different angles in order to reduce false alarms and possibly estimate the tumor size via registration of multiple final footprints around the tumor border.

It is worth noting that the proposed technique for detection of tumors is an extrapolation of laboratory-verified techniques and is applied to the problem of breast cancer sensing. The current work represents an early-stage exploration of this approach.

The remainder of the paper is organized as follows. In Section 2, we highlight some key properties of the controllable MTB actuators, which have been demonstrated experimentally by Martel et al. [6, 7]. We also present the general principle of tumor sensing and diagnosis. In Section 3, we describe possible schemes to detect and localize a breast tissue malignancy and estimate its size through the DMI-trackable bacterial propulsion-and-steering platform. In Section 4, we study the feasibility of the proposed strategies using an anatomically realistic breast model from the University of Wisconsin Computational Electromagnetics (UWCEM) Laboratory's Breast Phantom Repository [13]. Finally, we conclude with discussion on the potential and future extension of our approach in Section 5.

\section{Bacterial Microbots for Direct Tumor Targeting}

2.1. Main Characteristics of MTB. A flagellated MTB can be considered as a sophisticated cargo carrier with an embedded control interface, which comprises the following components as illustrated in Figure 1: (i) a steering wheel controlling

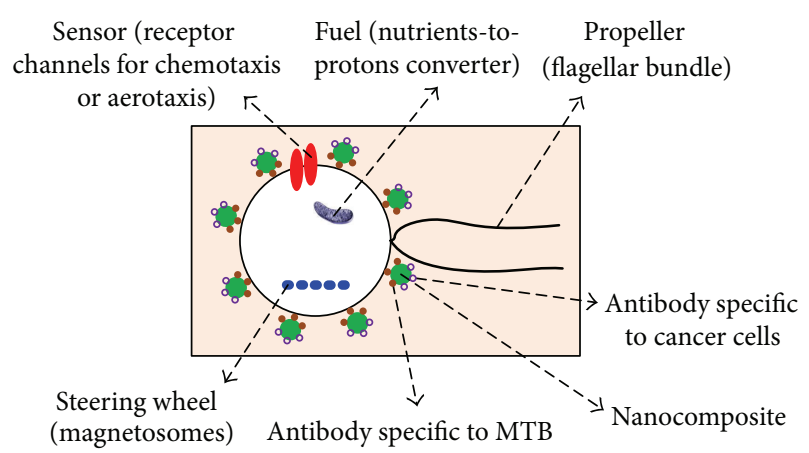

FIgURE 1: Key functional elements of MTB.

remotely the direction in which the MTB move (i.e., a chain of magnetosomes imparting to the MTB a magnetic moment so that the bacterium can align itself to an external magnetic field); (ii) propellers as the propulsion engines accelerating the MTB to move towards a targeted destination (i.e., two flagellar bundles allowing the bacterium to swim in a medium); (iii) a fuel unit powering all the components by scavenging energy from external sources (i.e., a nutrients-toprotons converter); and (iv) sensors acting as the interface between the environment and the MTB (i.e., receptor channels for chemotaxis or aerotaxis to detect nutrient gradients).

As shown in the previous works by Martel et al. [6, 7, 1417], MTB exhibit the following desirable properties making them ideal controllable bacterial actuators for direct tumor sensing.

2.1.1. Speed and Size. Magnetotactic cocci cells (e.g., the MC-1 bacteria) can achieve an average velocity of $200 \mu \mathrm{m} / \mathrm{s}$ in human blood with a peak velocity of $300 \mu \mathrm{m} / \mathrm{s}$. This is much faster than many other flagellated bacteria $(30 \mu \mathrm{m} / \mathrm{s})$ and the synthetic artificial flagella in the form of nanocoils actuated by a rotating magnetic field $(4.6 \mu \mathrm{m} / \mathrm{s})$. The maximum velocity could be maintained even when the MTB were loaded with an average of 70 liposomes with an overall diameter exceeding $150 \mathrm{~nm}$, suggesting the possibility of attaching a wide variety of nanoscale elements to the surface of its cell. The cell of the MC-1 MTB is spherical in shape with a diameter of $\sim 2 \mu \mathrm{m}$ being approximately half the diameter of the smallest capillaries in human, making it ideal for transiting in the tiniest blood vessels.

2.1.2. Propulsion and Steering. The two flagellar bundles of the MC-1 MTB provide a thrust force exceeding $4 \mathrm{pN}$, which is much higher than other flagellated bacteria $(0.3-0.5 \mathrm{pN})$. When subject to a magnetic field higher than 4 Gauss, the directional motions of the MTB were mainly influenced by magnetotaxis (i.e., aligned to magnetic field lines) as compared to chemotaxis (i.e., moving towards or away from a chemical source) and aerotaxis (i.e., moving towards or away from air or oxygen), thus fully remotely controllable using appropriate electronic hardware and software.

2.1.3. Byproducts of Bacterial Degradation. The degradation byproducts of the nonpathogenic bacteria could have 
a cytotoxic effect at high concentration, which imposes a limit on the amount of MTB that could be present in the human body at any time. Preliminary results showed that the cell viability was higher than $90 \%$ for $80 \mu \mathrm{L}$ of bacteria solution $\left(5 \times 10^{4}\right.$ cells $/ 200 \mu \mathrm{L}$ in cell culture solution) in a $200 \mu \mathrm{L}$ well, indicating the noncytotoxic effect of the bacteria at this concentration.

On the other hand, initial experiments conducted in human blood at $37^{\circ}$ showed that the velocity $V$ (in $\mu \mathrm{m} / \mathrm{s}$ ) at a time $t$ (expressed in minutes) after the beginning of operations of the MC-1 MTB decreased according to

$$
V=0.09 t^{2}-8.10 t+V_{0}, \quad 0 \leq t \leq 40 \mathrm{~min},
$$

where $V_{0} \approx 200 \mu \mathrm{m} / \mathrm{s}$ is the initial average velocity of the MTB prior to being injected into blood. The MC-1 MTB would remain effective for at least 40 minutes, and the decrease in speed was probably caused by the relatively high temperature of blood. This is beneficial from the perspective of biocompatibility and safe in vivo operations but limits the time at which the MC-1 MTB could operate. As a rough estimate, the average distance that could be traveled by an effective MC-1 MTB in the human body is given by $\int_{0}^{40} V$. $60 \mathrm{~d} t \approx 21 \mathrm{~cm}$, which is sufficient for the application of breast cancer theranostics.

2.1.4. Loading and Targeting. The delivery of a contrast agent to a tumoral lesion requires transitions through capillary sections of human microvasculature. The loading strategy depicted in Figure 1 can utilize antibodies specific to MC-1 cells or another effective method based on chemical bounding (see, e.g., [6]). The nanocomposites such as CNTs or FNPs can be functionalized by means of proper molecular groups such as peptides or antibodies, which are able to bind to cancerous cell receptors.

In order to deliver sufficient amounts of contrast agent to a tumor, a cluster of MC-1 MTB can be deployed simultaneously. It has been demonstrated that an agglomeration of MC-1 cells could be controlled like a unified organism to swim along a predetermined path by an external computer [14]. Finally, it has been shown using an in vitro model that MTB guided by magnetic fields could penetrate inside a multicellular tumor spheroid. As many tumors contain vasculogenic mimicry [18], these channels should help MTB to be evenly distributed inside a tumor.

\subsubsection{Aggregation of MTB. A relatively weak magnetic gra-} dient could be generated towards an aggregation zone, whose size can easily be in the millimeter range. The boundary of the aggregation zone is equivalent to approximately 0.5 Gauss with the center at 0 Gauss. When outside the aggregation zone, the bacteria follow the line of magnetic field. When inside the zone, the MTB are no longer influenced by magnetotaxis and begin random motions. If the MTB exit the aggregation zone, the magnetic field forces the MTB to move back to the zone by magnetotaxis. To improve targeting effectiveness, various magnetic field modulation modes have been developed depending on the characteristics of the

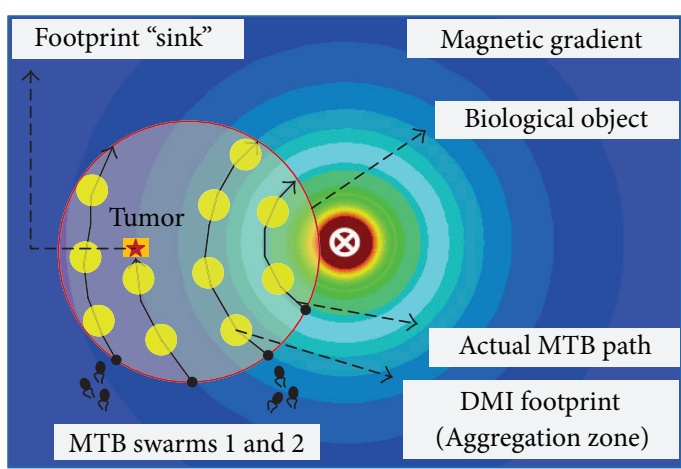

(a)

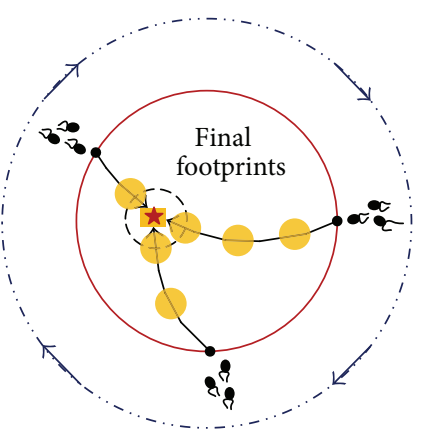

Targeting tumor from various angles

(b)

FIGURE 2: Illustration of (a) cancer detection and (b) examination with multiswarm MTB microbots.

angiogenesis network, that is, the capillary network feeding the tumor or the surrounding tissue. Once targeting has been completed, imaging could be performed to assess targeting efficacy and to confirm the region being affected. If needed, the operation can be repeated towards another targeted area by setting the aggregation zone accordingly.

2.2. Cancer Detection and Diagnosis with MTB Microbots. We illustrate the rationale for cancer detection and diagnosis with multiswarm MTB microbots in Figure 2(a), where two clumps of MTB are used as an example. Consider that these MTB are loaded with a microwave agent and injected simultaneously into the human body from two separate injection sites. An external source creates a magnetic field by means of a wire or coils placed close to the biological object under examination. As shown in Figure 2(a), we assume that the resulting magnetic field lines are concentric circles, which determine the direction in which the MTB will align. Apart from magnetotaxis, the MTB paths are mainly constrained by microvasculature networks in the breast. Hence, the movement of MTB is not directly affected by the heterogeneity within the breast.

We extend the DMI algorithm proposed in [11] to multiple targets as to be discussed in Section 3.2. This method is then utilized to track the motions of these two agglomerations of microbots by detecting and localizing the change in tissue dielectric properties in the targeted MTB aggregation zones 
as illustrated in Figure 2(a). Consequently, the measured DMI footprints corresponding to these aggregation zones result in snapshots of the actual MTB paths as illustrated in Figure 2(a). If a swarm of MTB microbots fails to detect a tumor (i.e., the corresponding microbot path does not cross the tumor), it will keep navigating towards the magnetic gradient until being maneuvered out of the human body. On the other hand, if the swarm senses a tumor, the contrast agent will be removed from the swarm and bound to cancer cell receptors. After load discharging, this swarm will become invisible to the DMI system, which only tracks variation in tissue dielectric properties caused by the agent. Hence, the location where the contrast agent finally accumulates will give rise to a "sink" on the preplanned MTB pathway as shown in Figure 2(a). Multiple tissue malignancies will result in several DMI footprint "sinks." Hence, tumors can be detected via this seeing-is-sensing principle.

On the other hand, we could inject MTB to target a detected and localized tumor from various directions as depicted in Figure 2(b). This can be achieved by using several magnetic field sources using, for example, direct current (dc) sources placed at different locations. These fields would guide MTB along distinct pathways to reach cancer cells from different angles. In this way, the final footprints immediately before a "sink" is observed would be scattered around the tumor edge. The tumor size which is related to the stage of breast cancer can thus be estimated via registration of all the final footmarks as demonstrated in Figure 2(b). This additional information will also help reduce false alarms for possible tumor locations. Hence, a tumor could be examined via this seeing-is-examination principle.

In the next section, we present possible strategies for the implementation of these approaches.

\section{Strategies for Breast Cancer Detection, Localization, and Size Estimation}

3.1. Proposed System Architecture and Protocol. To elaborate on our approaches, we consider a "heterogeneously dense" numerical phantom from the UWCEM breast model repository [13], which also includes one tumor having the median dielectric properties of malignant breast tissue [2]. Similar to $[8,11$, we employ the forty-element dipole antenna array arranged over five circular rings as shown in Figure 3(a). We plan to implement two-dimensional (2D) tumor sensing along the cross-sectional planes of the rings using threedimensional (3D) DMI data. For illustrative purpose, we focus on the third cross-sectional plane from the top, which slices through the tumor. The corresponding dielectric profile is shown in Figure 3(b). We apply the monostatic data obtained at the eight antennas of the third ring for tumor sensing. It is assumed that the presence of a contrast agent in a tissue region will substantially alter its dielectric properties [3]. As outlined in Section 2.2, our aim is to use the DMI algorithm to track the MTB swarms by sensing the induced changes in the surrounding tissue dielectric values. An important side result is the estimation of the breast average properties.
A block diagram representing the system architecture of breast tumor detection and diagnosis with multiswarm MTB microbots is shown in Figure 4. The basic sequence of events is detailed as follows.

3.1.1. Initialization. Prior to the injection of contrast-agentloaded microbots, we consecutively transmit ultra-wideband (UWB) probing waveforms from each antenna element as shown in Figure 3(b) and record the signals reflected from the breast at each element. These backscatter responses for the agent-free breast will be used for benchmarking during the DMI procedure at a later step.

3.1.2. Propulsion and Steering. We design the in-body microbot routes based on the breast geometry as well as the ROI in its interior and identify the corresponding injection sites on the breast surface. The ROIs may be acquired by using some preprocessing procedures (e.g., a noninvasive tomographic approach). The better the ROIs can be defined, the better the MTB paths can be planned. From this perspective, the system performance also depends on having a good-quality initialstage imaging system. The path planning should ensure that the entire ROI where cancers may be present is covered with sufficiently small intervals between neighboring paths to reduce the probability of miss. To reduce sensing time, we inject multiple swarms of agent-loaded microbots into the breast. Note that we do not produce the magnetic field in our simulations but assume that its effect is to guide the swarms along concentric magnetic field lines generated at one of the eight antennas for a specific interval $t_{\mathrm{PS}}$ until they reach the targeted aggregation zones as illustrated in Figure 3(b). During traveling to the aggregation zone, the microbots are not detected because they swim at different velocities and as such they disperse, making the density too low for tracking.

3.1.3. Tracking. Subsequently, we switch the system operational mode from "propulsion-and-steering" to "tracking." In order to set two aggregation zones simultaneously, we employ the following time-multiplexing scheme at a high frequency: $T_{\mathscr{M}_{1}} \rightarrow T_{\mathscr{M}_{2}} \rightarrow T_{\mathscr{A}_{1}} \rightarrow T_{\mathscr{M}_{1}} \rightarrow T_{\mathscr{M}_{2}} \rightarrow T_{\mathscr{A}_{2}} \rightarrow$ $\cdots$. During $T_{\mathscr{M}_{1}}$ and $T_{\mathscr{M}_{2}}$, two magnetic gradients are generated to set the two aggregation zones. Furthermore, during $T_{\mathscr{A}_{i}}(i=1,2, \ldots, 8)$, the waveform used in the initialization phase is retransmitted from the $i$ th antenna element, and the corresponding backscatter response is recorded. We then apply the DMI algorithm to track multiple swarms within their respective aggregation zones. The size of the aggregation zones is in the range of $2 \mathrm{~mm}$, which is much smaller than the cancerous lesion to be detected.

3.1.4. Decisionmaking. We then confirm whether the tumor sensing is completed, given the microbot footmarks and subject to a number of criteria as listed in Figure 4 . We need to check whether the swarms of microbots are still present in the breast, whether a DMI footprint "sink" has been observed, and whether all the preplanned paths have been completed. If all the answers are confirmative, we declare that tissue anomalies have been positively detected. Otherwise, 


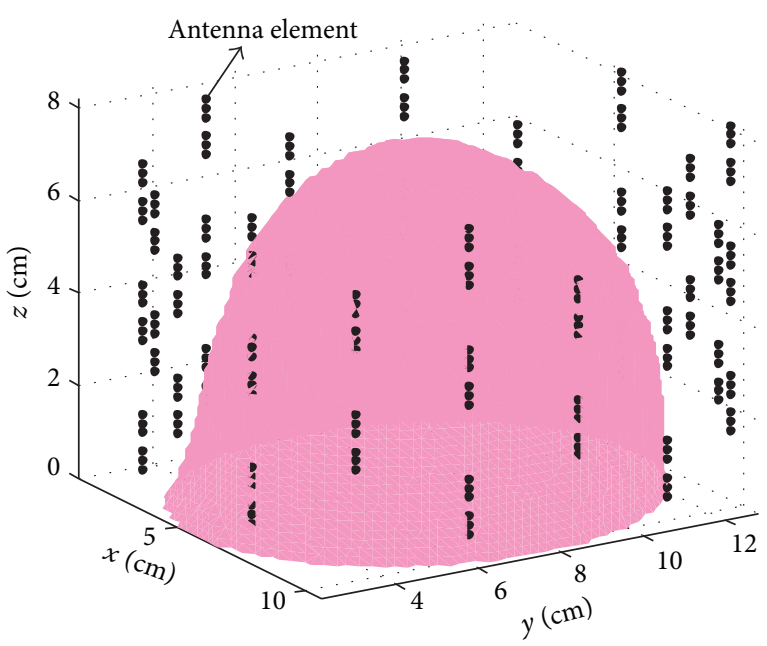

(a)

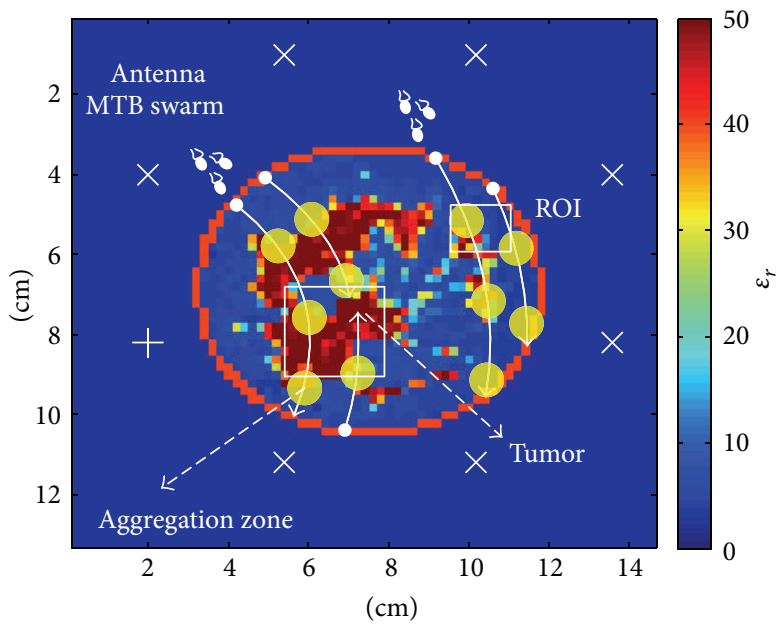

(b)

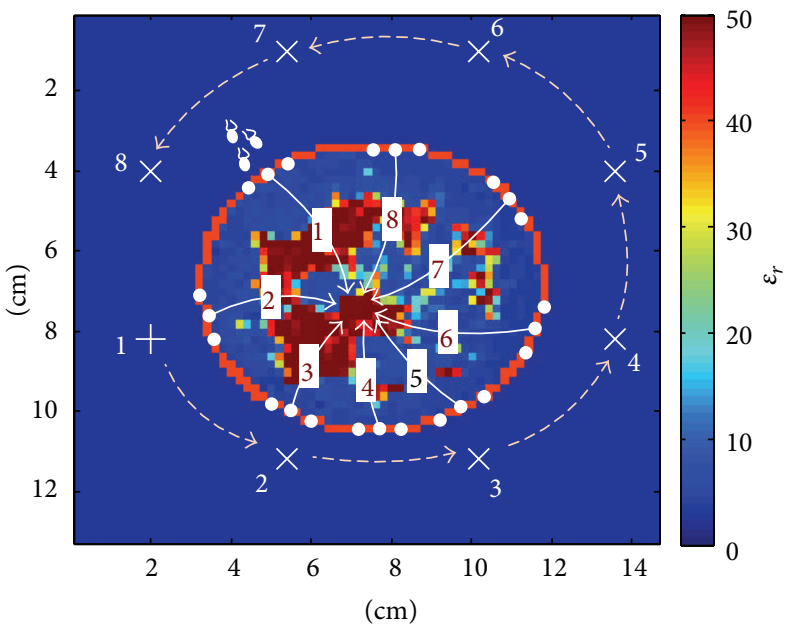

(c)

Figure 3: (a) Illustration of the tumor sensing and diagnosis system using a configuration similar to [8, 11]. (b) Dielectric profile at $1 \mathrm{GHz}$ for the coronal cross-section through the third antenna ring. We assume concentric magnetic field lines originating from a dc source via one of the antennas (marked with a "+") during the propulsion-and-steering phase. All the eight antennas probe the breast sequentially with UWB pulses during the tracking phase after the MTB reach the targeted aggregation zones (marked with yellow circles). Two clumps of MTB are injected simultaneously into the breast. (c) Size estimation of the tumor by targeting it from different angles; the eight antennas are sequentially employed to carry a dc for generation of magnetic fields.

the system may inject new agglomerations of microbots or continue maneuvering the old ones, depending on whether the previously deployed swarms are still in the breast. It is worth noting that all the preplanned microbot trajectories should be completed even if a tumor has been detected. This is to ensure successful detection and targeting of multiple cancerous masses.

3.1.5. Multidirectional Targeting. Provided with the a priori knowledge of the tumor location acquired in the previous steps, we then replan the microbot paths and injection sites in order to target the tumor from different angles as demonstrated in Figure 3(c). The survey plan should ensure that these angles-of-arrival are evenly distributed between 0 and $2 \pi$ to facilitate a full 360 -degree view of the tumor. A possible strategy is shown in Figure 3(c), where each antenna (indexed from 1 to 8 ) takes turn to carry a dc for generation of magnetic fields. Suppose that we have extracted the rough position of a tumor through the previous operations. For each dc excitation, we can identify a few adjacent infusion sites on the breast surface as shown in Figure 3(c). These sites are selected such that theoretical microbot paths originated from them may intersect at the tumor. For illustrative purposes, we draw one path (marked with the antenna index) for each antenna as depicted in Figure 3(c). We then administer multiswarm MTB microbots and perform propulsion, steering, and tracking until all the "sinks" are observed. If the distance between two successive footprints is sufficiently small, the final DMI footprints provide markers on the tumor edge and therefore could be used to approximate its size. 


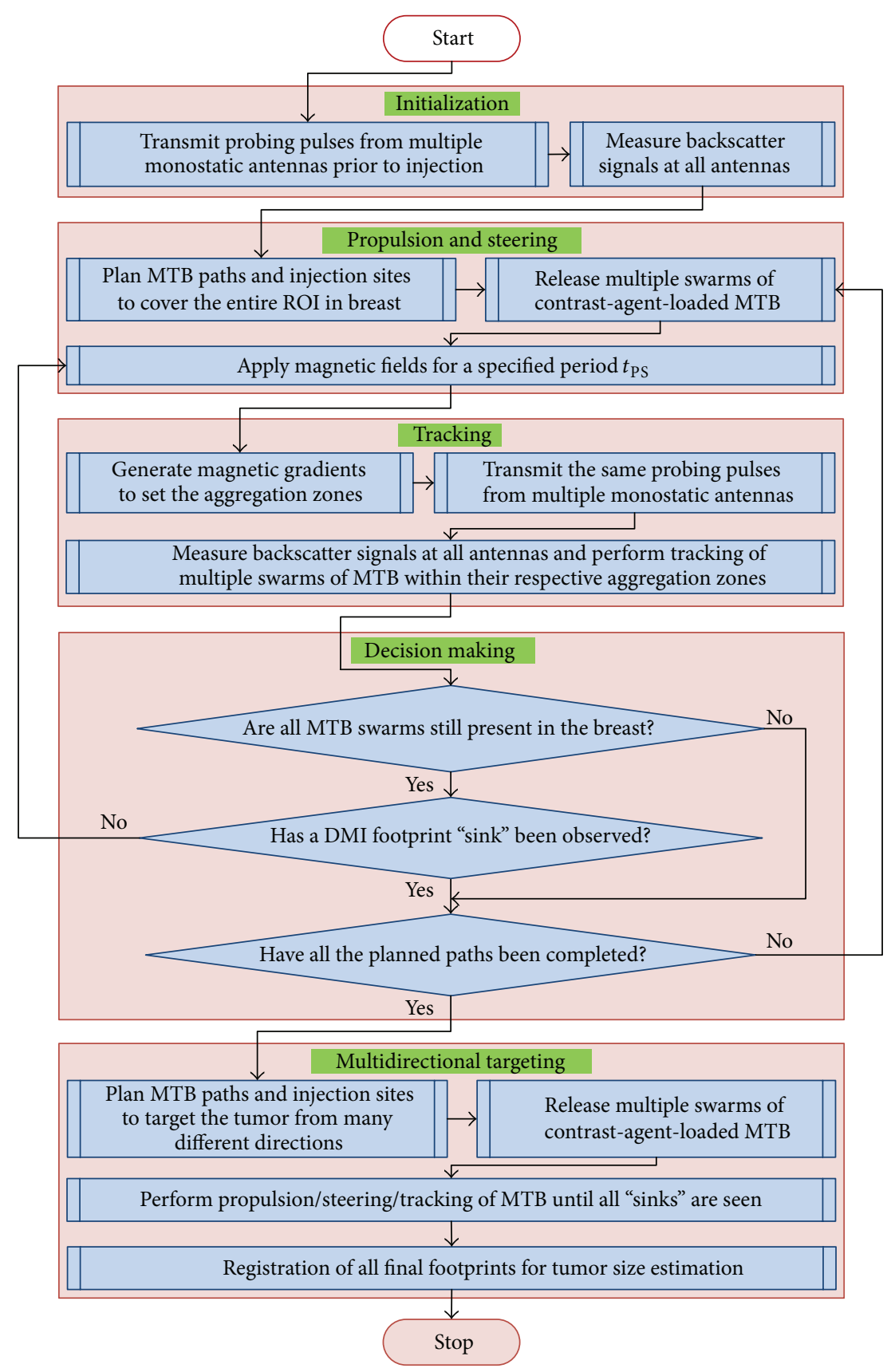

FIGURE 4: Flowchart representing the system architecture of breast tumor detection and diagnosis with multiswarm MTB microbots.

This additional step also helps reduce false alarms for tumor detection and localization by exploiting the microbot path diversity.

3.2. Tracking of Multiple Swarms of MTB Microbots. In this section, we present a novel DMI algorithm to detect multiple local dielectric variations due to multiswarm contrast-agentloaded MTB microbots. We can summarize the principles of the algorithm as follows. A microwave contrast agent such as CNTs or FNPs loaded onto multiple aggregations of MTB changes the local dielectric properties of the tissue regions where the MTB are present. We can extract the difference between the backscatter responses before and after the injection of MTB over time. We then formulate the problem from the perspective of signal model selection (differential signal corrupted by noise versus noise alone) and apply the information theoretic criterion such as the Akaike information criterion or the minimum description length to reliably estimate the locations of the induced dielectric changes [11].

We consider a cluttered breast medium as shown in Figure 5. Two swarms of MTB microbots are present in the interference-prone biological environment with a large number of normal breast tissue inhomogeneities. Consider 


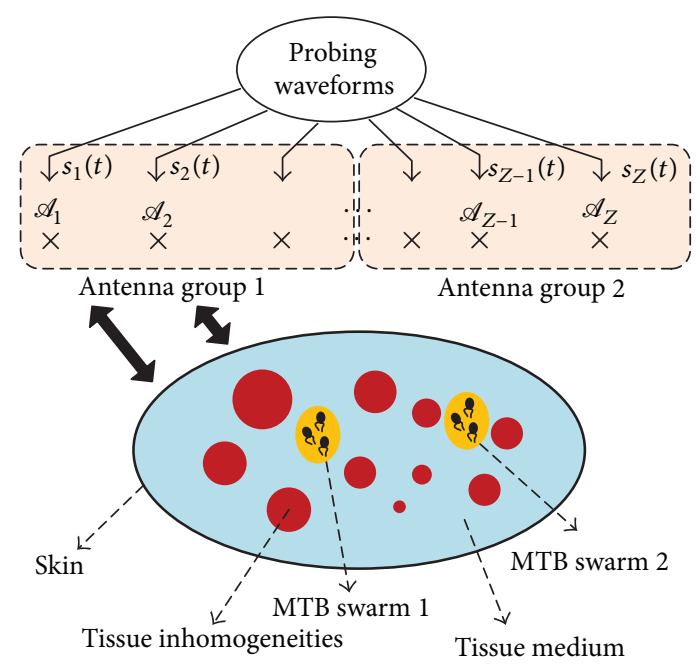

FIGURE 5: An interference-prone breast channel where two agentloaded MTB swarms are present in the cluttered breast with tissue inhomogeneities. A radar system containing multiple spatially diverse monostatic antenna elements is deployed for tracking the swarms.

a radar system comprising multiple monostatic antenna elements, $\mathscr{A}_{1}, \mathscr{A}_{2}, \ldots, \mathscr{A}_{Z}$. Each antenna represents both a source and a sensor of the electromagnetic signals. We will use the subscript $z$ in all the following analyses to indicate the quantities associated with antenna $\mathscr{A}_{z}(z=1,2, \ldots, Z)$. Let $s_{z}(\cdot)$ and $o_{z}(\cdot)$ denote, respectively, the discrete-time transmitted and received signals by $\mathscr{A}_{z}$. The following general relationship can be obtained:

$$
o_{z}(m)=\sum_{l=0}^{L_{z}-1} h_{z}(l) s_{z}(m-l)+u_{z}(m)
$$

where $h_{z}(\cdot)$ is the discrete channel response, $u_{z}(\cdot)$ is the additive white Gaussian noise (AWGN), and $L_{z}$ denotes the order of the discrete channel. Equation (2) can be rewritten in the matrix form as

$$
\mathbf{o}_{z}=\mathbf{H}_{z} \mathbf{s}_{z}+\mathbf{u}_{z}
$$

where

$$
\begin{gathered}
\mathbf{o}_{z}=\left[o_{z}\left((n-1)\left(L_{z}+M_{z}-1\right)+1\right) \cdots o_{z}\left(n\left(L_{z}+M_{z}-1\right)\right)\right]^{T}, \\
\mathbf{H}_{z}=\left[\begin{array}{cccc} 
& & h_{z}(0) \\
& h_{z}(0) & h_{z}(1) \\
& \vdots & \vdots & \vdots \\
h_{z}(0) & h_{z}(1) & \cdots & h_{z}\left(M_{z}-1\right) \\
h_{z}(1) & h_{z}(2) & \cdots & h_{z}\left(M_{z}\right) \\
\vdots & \vdots & \vdots & \\
h_{z}\left(L_{z}-2\right) & h_{z}\left(L_{z}-1\right) & & \\
h_{z}\left(L_{z}-1\right) & &
\end{array}\right],
\end{gathered}
$$

$$
\begin{gathered}
\mathbf{s}_{z}=\left[s_{z}\left(n M_{z}\right) \cdots s_{z}\left((n-1) M_{z}+1\right)\right]^{T} \\
\mathbf{u}_{z}=\left[u_{z}\left((n-1)\left(L_{z}+M_{z}-1\right)+1\right) \cdots u_{z}\left(n\left(L_{z}+M_{z}-1\right)\right)\right]^{T} .
\end{gathered}
$$

In (4), $n=1,2, \ldots, N$ is the index of each observation. $\mathbf{o}_{z}, \mathbf{H}_{z}$, $\mathbf{s}_{z}$, and $\mathbf{u}_{z}$ are the $\left(L_{z}+M_{z}-1\right) \times 1$ observation vector, the $\left(L_{z}+M_{z}-1\right) \times M_{z}$ channel matrix, the $M_{z} \times 1$ source signal vector, and the $\left(L_{z}+M_{z}-1\right) \times 1$ noise vector, respectively. The symbol $T$ denotes matrix transpose.

Suppose that the delay taps introduced by these two swarms of agent-attached microbots are $\ell_{1, z}$ and $\ell_{2, z}(0<$ $\left.\ell_{1, z}, \ell_{2, z} \leq L_{z}-1\right)$. The presence of contrast media in the breast will distort the biological channel response by inducing changes in the tissue dielectric properties. We note that only late responses with delays greater than $\mathscr{L}_{z}=\min \left\{\ell_{1, z}, \ell_{2, z}\right\}$ will be affected. Hence, the new channel matrix after injection of MTB is

$$
\widetilde{\mathbf{H}}_{z}=\mathbf{H}_{z}+\Delta \mathbf{H}_{z}
$$

where the $(i, j)$ th $\left(i=1,2, \ldots, L_{z}+M_{z}-1 ; j=1,2, \ldots, M_{z}\right)$ entry of the perturbation matrix $\Delta \mathbf{H}_{z}$ is expressed as

$$
\Delta \mathbf{H}_{z}[i, j]= \begin{cases}0 ; & \text { if } \mathbf{H}_{z}[i, j]=0 \\ 0 ; & \text { if } \mathbf{H}_{z}[i, j]=h_{z}(l) \text { and } l<\mathscr{L}_{z} \\ \Delta h_{z}(l) ; & \text { if } \mathbf{H}_{z}[i, j]=h_{z}(l) \text { and } l \geq \mathscr{L}_{z}\end{cases}
$$

In (6), $\Delta h_{z}(l)$ is a nonzero term summarizing the overall perturbation effect introduced to the $l$ th tap of the biological channel.

Subsequently, we can split all antennas into two groups as shown in Figure 5. For each member in antenna group $k(k=1,2), \mathscr{L}_{z}=\ell_{k, z}$, and each group only detects its nearest microbot swarm. As a result, we can apply readily the information-theoretic-criteria-based DMI algorithm in [11] for single-target localization, where the time-of-arrival (TOA) of the backscatter from the swarm, $\mathscr{L}_{z}$, is estimated. If the microbot paths are planned such that the two swarms are well separated in the breast at all time instants, it is straightforward to perform antenna grouping, knowing the locations of the injection sites and the dc source.

3.3. TOA Data Fusion for Unknown Average Tissue Dielectric Properties. To estimate the cancer location, the TOAs measured at different antennas can be combined by using the data fusion method proposed in [19]. This algorithm, however, requires knowledge about the average tissue dielectric properties, which is very difficult to obtain in a reallife clinical setting. The current tumor sensing strategy offers an interesting way to overcome the above challenge by estimating both the average wave propagation speed and the swarm location in the breast. We assume that an antenna $\mathscr{A}_{z_{k}}(k=1,2)$ is tracking the $k$ th microbot swarm. $\mathscr{A}_{z_{k}}$ is located at $\left(x_{z_{k}}, y_{z_{k}}\right)$ and measures a TOA $\tau_{z_{k}}=\mathscr{L}_{z_{k}} T_{0}$, where $T_{0}$ is the sampling time. The TOA $\tau_{z_{k}}$ defines a circle centered at $\mathscr{A}_{z_{k}}$ with radius $r_{z_{k}}=v \tau_{z_{k}} / 2$, where $v$ is the average speed of electromagnetic waves in the breast medium and is related to 
the mean tissue permittivity $\varepsilon_{b}$ and conductivity $\sigma_{b}$ through the following relationship:

$$
v=\frac{1}{\sqrt{\left(\mu_{0} \varepsilon_{b} / 2\right)\left[\sqrt{1+\left(\sigma_{b} / \omega \varepsilon_{b}\right)^{2}}+1\right]}} .
$$

As knowledge of the TOA estimation error statistics is not available, we locate the $k$ th microbot swarm by minimizing the sum of square of the distances from the swarm to the points of intersection of the range circles closest to it. The objective function is given by

$$
\widehat{\mathbf{x}}_{k}=\arg \min _{\mathbf{x}_{k}}\left\{\sum_{j_{k}=1}^{J_{k}}\left\|\mathbf{x}_{k}-\overline{\mathbf{x}}_{j_{k}}\right\|^{2}\right\} \text {. }
$$

The parameter $J_{k}$ is the total number of intersection points. $\mathbf{x}_{k}=\left[\mathbf{x}_{k, 0}^{T}, \delta_{1}, \ldots, \delta_{Z_{k}}\right]^{T}$, where $\mathbf{x}_{k, 0}=\left[x_{k}, y_{k}\right]^{T}$ is the true position of the $k$ th swarm, $\delta_{z_{k}}$ represents the value of distance measurement error between $\mathscr{A}_{z_{k}}$ and the swarm, and $Z_{k}$ is the cardinality of antenna group $k \cdot \overline{\mathbf{x}}_{j_{k}}=\left[\overline{\mathbf{x}}_{0, j_{k}}^{T}, 0, \ldots, 0\right]^{T}$, where $\overline{\mathbf{x}}_{0, j_{k}}=\left[\bar{x}_{j_{k}}, \bar{y}_{j_{k}}\right]^{T}$ is the $j_{k}$ th intersection.

Let $\mathbf{c}_{k}=\left(1 / J_{k}\right) \sum_{j_{k}=1}^{J_{k}} \overline{\mathbf{x}}_{0, j_{k}}$ be the center of all intersection points, and let $d_{z_{k}}\left(\mathbf{c}_{k}\right)=\sqrt{\left(x_{z_{k}}-x_{\mathbf{c}_{k}}\right)^{2}+\left(y_{z_{k}}-y_{\mathbf{c}_{k}}\right)^{2}}$ be the distance between $\mathscr{A}_{z_{k}}$ and $\mathbf{c}_{k}=\left[x_{\mathbf{c}_{k}}, y_{\mathbf{c}_{k}}\right]^{T}$. Given an average speed of waves $v$, an optimum noniterative solution of (8) can be written as [19]

$$
\widehat{\mathbf{x}}_{k}(v)=\boldsymbol{\Omega}_{k}^{T}\left(\boldsymbol{\Omega}_{k} \boldsymbol{\Omega}_{k}^{T}\right)^{-1}\left(\mathbf{r}_{k}-\mathbf{d}_{k}\right)+\frac{1}{J_{k}} \sum_{j_{k}=1}^{J_{k}} \overline{\mathbf{x}}_{j_{k}},
$$

where

(i) $\boldsymbol{\Omega}_{k}=\left[\begin{array}{ccccc}\left(x_{\mathfrak{c}_{k}}-x_{1}\right) / d_{1}\left(\mathbf{c}_{k}\right) & \left(y_{c_{k}}-y_{1}\right) / d_{1}\left(\mathbf{c}_{k}\right) & 1 & \cdots & 0 \\ \vdots & \vdots & \vdots & \ddots & \vdots \\ \left(x_{\mathfrak{c}_{k}}-x_{Z_{k}}\right) / d_{Z_{k}}\left(\boldsymbol{c}_{k}\right) & \left(y_{c_{k}}-y_{Z_{k}}\right) / d_{Z_{k}}\left(\mathfrak{c}_{k}\right) & 0 & \cdots & 1\end{array}\right]$,

(ii) $\mathbf{r}_{k}=\left[r_{1}, \ldots, r_{Z_{k}}\right]^{T}$ is the range measurements vector; and

(iii) $\mathbf{d}_{k}=\left[d_{1}\left(\mathbf{c}_{k}\right), \ldots, d_{Z_{k}}\left(\mathbf{c}_{k}\right)\right]^{T}$ is the distance vector.

The detailed derivation of (9) can be found in [20].

Finally, we enforce the constraint that DMI footprints should be roughly aligned with the theoretical circular magnetic field with radius $R$ generated by a dc source located at $\mathbf{x}_{\mathrm{dc}}=\left[x_{\mathrm{dc}}, y_{\mathrm{dc}}\right]^{T}$. As a result, the average speed $v$ is estimated to be

$$
v^{*}=\arg \min _{v}\left|\left\|\widehat{\mathbf{x}}_{k}(v)-\mathbf{x}_{\mathrm{dc}}\right\|-R\right|
$$

and the corresponding swarm footprint is given by $\widehat{\mathbf{x}}_{k}\left(v^{*}\right)$.

\section{Simulation Results}

We use several numerical examples to elaborate on the aforementioned cancer detection and diagnosis methods. Specifically, we simulate possible movements of the swarms due to the presumed magnetic field and test the ability of the DMI algorithm for tracking the microbot trajectories and sensing the tumor. Figure 3 provides the layout of the microbotsassisted diagnostic system as discussed in Section 3. The injection sites are spaced on the circumference of the crosssection of the numerical phantom shown in Figure 3(b), which is also illustrated in Figure 6(a) (marked with circles). Figure 6(a) also shows the preplanned microbot paths covering uniformly the two ROIs in the breast interior. The gap between two neighboring pathways is $\sim 5 \mathrm{~mm}$, which gives rise to the resolution of the specific survey plan. Two microbot swarms are injected into the breast simultaneously, where their injection sites are spaced apart during the whole sensing process to ensure reliable tracking as discussed in Section 3.2. We assume that each swarm of microbots follows a directional random walk during the propulsionand-steering stage. Thus, each step size fluctuates according to an exponential probability density function (pdf) with a mean value $\beta$. Furthermore, the direction at each step is uniformly distributed between $[\varphi-\theta, \varphi+\theta]$. The parameter $\varphi$ is the average direction towards the magnetic gradient and $\theta$ characterizes the effect of directional disturbance due to other gradients such as chemotaxis and aerotaxis as well as random microvasculature structures. Finally, we assume that the microbots in the aggregation zones follow an isotropic random walk with the same step size distribution as the one used in the tracking stage, which has been demonstrated experimentally $[6,7,14-17]$.

The initial velocities of both swarms are assumed to be $200 \mu \mathrm{m} / \mathrm{s}$, which decrease with time after being injected into the breast according to (1). The duration for the propulsionand-steering phase is $t_{\mathrm{PS}}=2 \mathrm{~min}$. To synthesize the effect of contrast-agent-loaded microbots on the tissue dielectric properties, we adopt CNTs as the agent to modify the dielectric properties for one $2 \times 2 \times 2 \mathrm{~mm}^{3}$ cubic voxel inside the breast phantom. We assume that the relative permittivity and conductivity of this voxel are $22 \%$ and $70 \%$ higher than the median properties of malignant breast tissue, following the measurement data in [8].

Each dipole antenna in Figure 6(a) is driven with a modulated Gaussian pulse having a bandwidth covering 0.5 to $3.5 \mathrm{GHz}$, and the backscatter signal is recorded at the same antenna (monostatic configuration). The signal-tonoise ratio (SNR) used in our simulation study is $10 \mathrm{~dB}$.

Figure 6(b) shows a typical simulated sensing process $\left(\theta=30^{\circ}, \beta=100 \mu \mathrm{m}\right)$ and plots the associated DMI footprints. Samples of the exact locations of microbots during the tracking phase are marked with triangles, and the corresponding location estimates are marked with squares. For example, consider two swarms injected simultaneously from the 1 st and the 4 th injection sites. These two agglomerations of microbots were directed by the magnetic fields to swim for $2 \mathrm{~min}$ and reach the first aggregation zones where the tracking was performed. Once the tracking is completed, the system mode was switched back to propulsion-and-steering. The swarms continued navigating and the DMI algorithm presented in Section 3.2 was applied to locate the microbots for the earlier tracking cycle. The same steps were repeated 


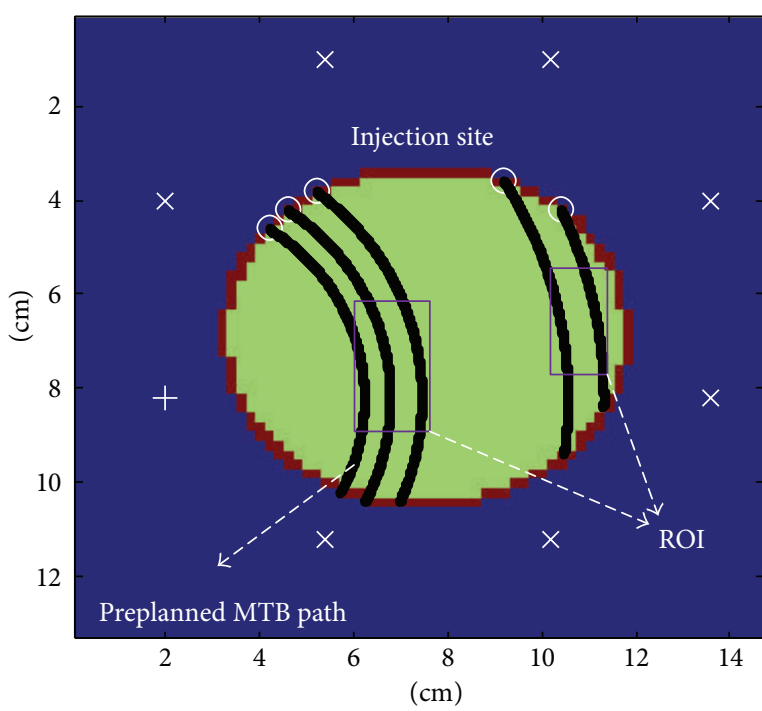

(a)

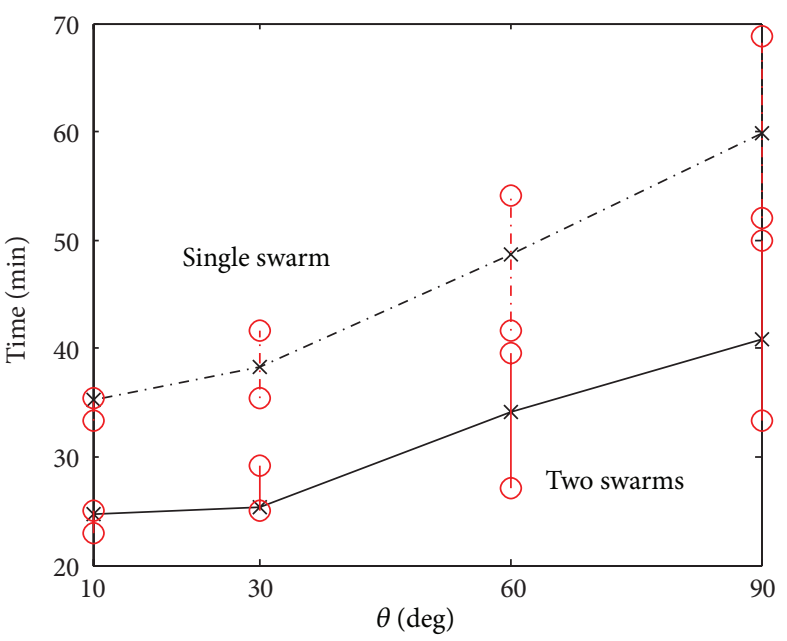

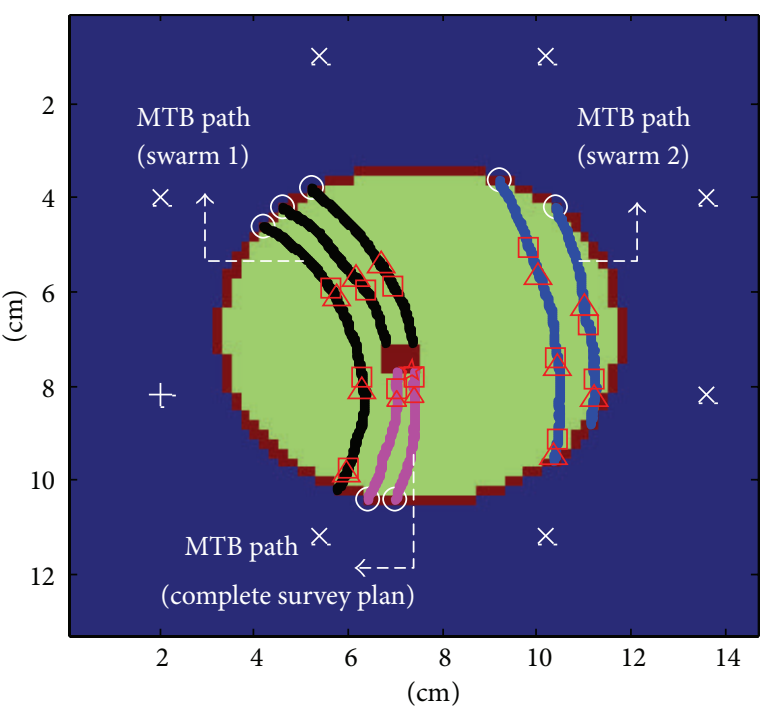

(b)

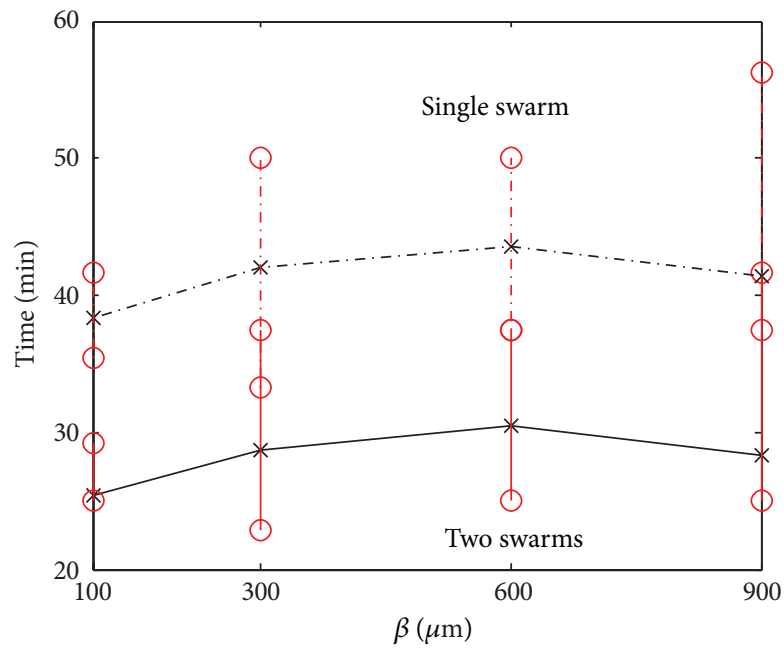

(c)

Figure 6: (a) Preplanned MTB paths for the system setup in Figure 3(b). (b) Simulated DMI footprints. The MTB injection sites are marked with "о." The actual and estimated positions of the MTB during the tracking phase are marked with " $\triangle$ " and " $\square$," respectively. The estimated final destination of contrast agent (i.e., DMI footprint "sink") is marked with " $\star$." (c) Sensing time versus angular spread $\theta$ and mean step size $\beta$ of the directional random walk for single- and two-swarm administration. The fixed parameter values are $\beta=100 \mu \mathrm{m}$ in the former and $\theta=30^{\circ}$ in the latter. The markers " $x$ " and " $"$ " correspond to the sensing time mean and dispersion, respectively.

periodically. When any of the two swarms left the breast, we injected a new agglomeration of microbots from the next injection site, and so on. The overall good agreement between the estimated and exact microbot locations demonstrates the feasibility of the proposed DMI algorithm for tracking the CNTs-loaded MTB microbots. Ideally, a DMI footprint "sink" indicating the cancer location will be recorded, as described in Section 3. Finally, two additional microbot injections at the bottom of the breast are required to complete the original path plan shown in Figure 6(a).

The angular spread $\theta$ and the mean step size $\beta$ of the random walk process will affect the cancer diagnostic time, which is an important system performance metric. Figure 6(c) shows the mean, maximum, and minimum tumor sensing times for various $\theta$ and $\beta$ by carrying out ten independent simulations of the microbot tracking process. Apparently, by exploiting two swarms of microbots in parallel, remarkable reduction in the time spent in completing the breast examination is achieved as compared to the singleswarm case. Moreover, the mean and dispersion of the sensing time increase with the angular spread $\theta$, while the average sensing duration is not affected by different $\beta$ values though its dispersion increases with $\beta$ in general.

Finally, Figure 7(a) illustrates a typical multidirectional tumor targeting process based on the strategy presented in Section 3.1. For each dc source, only one injection site is designated in order to send an MTB swarm to target the tumor location from one particular direction. We also reduce 


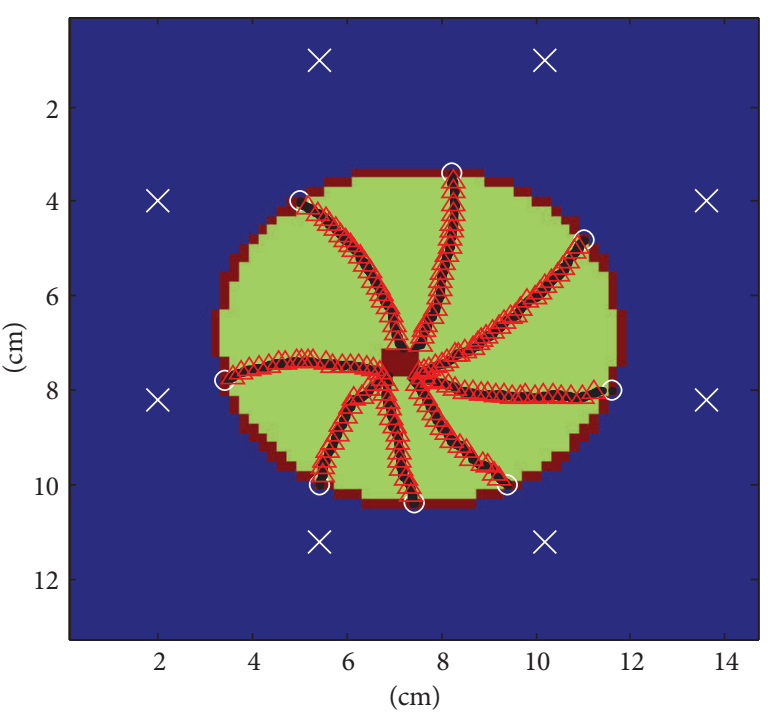

(a)
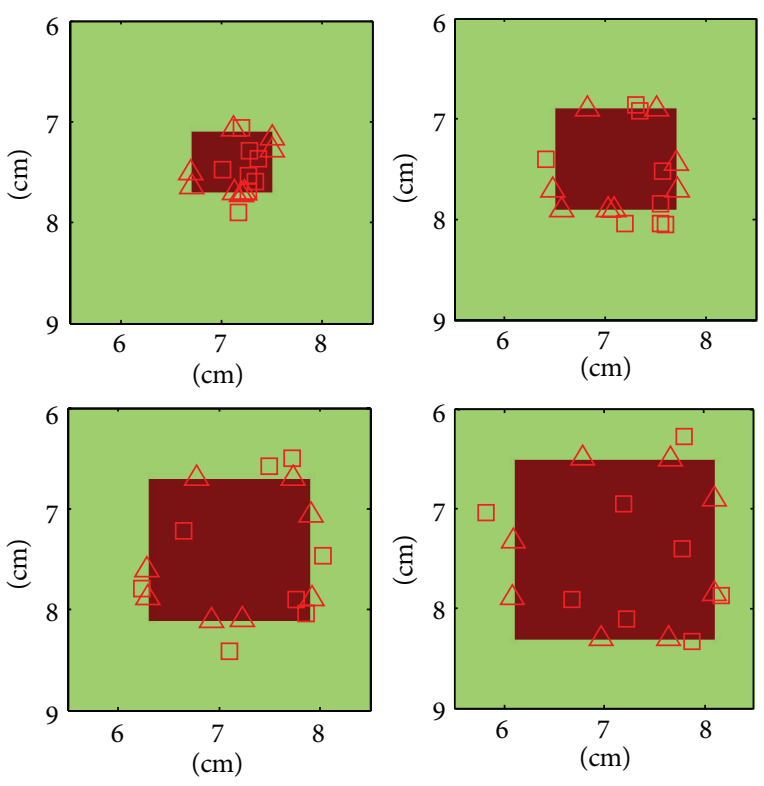

(b)

Figure 7: (a) Multidirectional tumor targeting. (b) Simulated DMI footprints for four tumors with different sizes. The actual and estimated final MTB footprints are marked with “ $\triangle$ " and “ $\square$ ", respectively.

the propulsion-and-steering period to $10 \mathrm{~s}$ to ensure a more frequent tracking process. Subsequently, Figure 7(b) demonstrates the final footprints of MTB swarms for four tumors with varying sizes. As can be seen from this figure, the locations of these footprints correlate well with the size of the tumor, which demonstrates the potential of the proposed method for tumor size estimation.

\section{Conclusion}

This preliminary study presented a novel scheme for microwave breast cancer detection and diagnosis with the use of multiswarm bacterial microbots. The proposed technique is more accurate and is inherently integrated with the followup drug delivery and cancer treatment as compared to other noninvasive imaging modalities such as X-ray, magnetic resonance imaging (MRI), ultrasound scan, and microwave imaging. However, it is minimally invasive due to the injection procedure of MTB. Moreover, compared to MRI-guided tumor targeting based on MTB [14], the current approach is much cheaper by replacing the expensive MRI equipment with the low-cost microwave system.

Several key assumptions must be verified for these tactics to be clinically feasible. Firstly, the efficiency of the MTB as controlled trackable propulsion and steering systems, as well as the ability of our proposed DMI algorithm to detect the microbot swarms based on the effect of their contrast agent cargos inside the tissue should be confirmed. The complex permittivity change due to the agent-attached swarms will depend on many factors and must be studied further; the key issue for the feasibility of our approach is that they induce a change in the dielectric properties which can be detected by our DMI technique. Secondly, due to the physiological environment (microvasculature, tissue environment, etc.), it is more likely that the maximum density of microbots in the aggregation zone will be lower than the one achieved in free space such as in water. As such, the sensitivity of the DMI system for tracking microbots in the aggregation zone should be validated experimentally. Finally, the immune response of MTB will be investigated further (e.g., development of mutation of the same specie of MTB for repeated operation within a short duration).

There are many ways for improving and expanding the capabilities of the proposed system. For example, to better plan the swarm trajectories and ensure a good coverage of the breast interior, a priori information could be used from a preprocessing step of microwave tomography using the same configuration. In addition, further postprocessing using the differential backscatter data could infer additional information about the lesion morphology and avoid false detection [9], which could occur, for example, if the agent is falsely unloaded. Multimodality imaging including MRI will also be considered. Finally, microwave hyperthermia treatment could be performed once the tissue malignancy is localized and the contrast agent is successfully transported to the tumor site thereby improving its heating response [3].

\section{Conflict of Interests}

The authors declare that there is no conflict of interests regarding the publication of this paper.

\section{Acknowledgment}

This research was partly supported by 2013 Guangdong Natural Science Funds for Distinguished Young Scholar (Project ID: S2013050014223).

\section{References}

[1] N. K. Nikolova, "Microwave imaging for breast cancer," IEEE Microwave Magazine, vol. 12, no. 7, pp. 78-94, 2011. 
[2] M. Lazebnik, D. Popovic, L. McCartney et al., "A large-scale study of the ultrawideband microwave dielectric properties of normal, benign and malignant breast tissues obtained from cancer surgeries," Physics in Medicine and Biology, vol. 52, no. 20, pp. 6093-6115, 2007.

[3] A. Mashal, B. Sitharaman, X. Li et al., "Toward carbonnanotube-based theranostic agents for microwave detection and treatment of breast cancer: enhanced dielectric and heating response of tissue-mimicking materials," IEEE Transactions on Biomedical Engineering, vol. 57, no. 8, pp. 1831-1834, 2010.

[4] S. Semenov, N. Pham, and S. Egot-Lemaire, "Ferroelectric nanoparticles for contrast enhancement microwave tomography: feasibility assessment for detection of lung cancer," in Proceedings of the World Congress on Medical Physics and Biomedical Engineering, pp. 311-313, Munich, Germany, September 2009.

[5] A. I. Minchinton and I. F. Tannock, "Drug penetration in solid tumours," Nature Reviews Cancer, vol. 6, pp. 583-592, 2006.

[6] S. Martel, M. Mohammadi, O. Felfoul, Z. L. Zhao Lu, and P. Pouponneau, "Flagellated magnetotactic bacteria as controlled MRI-trackable propulsion and steering systems for medical nanorobots operating in the human microvasculature," International Journal of Robotics Research, vol. 28, no. 4, pp. 571-582, 2009.

[7] N. Mokrani, O. Felfoul, F. A. Zarreh et al., "Magnetotactic bacteria penetration into multicellular tumor spheroids for targeted therapy," in Proceedings of the 32nd Annual International Conference of the IEEE Engineering in Medicine and Biology Society (EMBC '10), pp. 4371-4374, Buenos Aires, Argentina, September 2010.

[8] J. D. Shea, P. Kosmas et al., "Contrast-enhanced microwave imaging of breast tumors: a computational study using 3D realistic numerical phantoms," Inverse Problems, vol. 26, Article ID 074009, 2010.

[9] Y. Chen, I. J. Craddock, and P. Kosmas, "Feasibility study of lesion classification via contrast-agent-aided UWB breast imaging," IEEE Transactions on Biomedical Engineering, vol. 57, no. 5, pp. 1003-1007, 2010.

[10] P. Kosmas and Y. Chen, "Possibilities for microwave breast tumor sensing via contrast-agent-loaded nanorobots," in Proceedings of the 6th European Conference on Antennas and Propagation (EUCAP '12), Prague, Czech Republic, March 2012.

[11] Y. Chen and P. Kosmas, "Detection and localization of tissue malignancy using contrast-enhanced microwave imaging: exploring information theoretic criteria," IEEE Transactions on Biomedical Engineering, vol. 59, no. 3, pp. 766-776, 2012.

[12] Y. Chen and P. Kosmas, "Microwave breast tumor sensing and targeting using multiswarm contrast-agent-loaded bacterial nanorobots," in Proceedings of the International Conference on Electromagnetics in Advanced Applications (ICEAA '12), Cape Town, South Africa, September 2012.

[13] E. Zastrow, S. K. Davis, M. Lazebnik, F. Kelcz, B. D. Van Veen, and S. C. Hagness, "Development of anatomically realistic numerical breast phantoms with accurate dielectric properties for modeling microwave interactions with the human breast," IEEE Transactions on Biomedical Engineering, vol. 55, pp. 2792-2800, 2008.

[14] S. Martel, O. Felfoul, J.-B. Mathieu et al., "MRI-based medical nanorobotic platform for the control of magnetic nanoparticles and flagellated bacteria for target interventions in human capillaries," International Journal of Robotics Research, vol. 28, no. 9, pp. 1169-1182, 2009.
[15] S. Martel, O. Felfoul, M. Mohammadi, and J. B. Mathieu, "Interventional procedure based on nanorobots propelled and steered by flagellated magnetotactic bacteria for direct targeting of tumors in the human body (IEEE EMBC '08)," in Proceedings of the Annual International Conference of the IEEE Engineering in Medicine and Biology Society, Vancouver, Canada, August 2008.

[16] S. Martel, O. Felfoul, and M. Mohammadi, "Flagellated bacterial nanorobots for medical interventions in the human body," in Proceedings of the 2nd Biennial IEEE/RAS-EMBS International Conference on Biomedical Robotics and Biomechatronics (BioRob '08), pp. 264-269, October 2008.

[17] C. C. Tremblay, J. Jean, L. Marchand et al., "Robotic platform for real-time tracking of a single fast swimming bacterium," in Proceedings of the International Symposium on Optomechatronic Technologies (ISOT '10), Toronto, Canada, October 2010.

[18] R. Folberg, M. J. C. Hendrix, and A. J. Maniotis, "Vasculogenic mimicry and tumor angiogenesis," The American Journal of Pathology, vol. 156, no. 2, pp. 361-381, 2000.

[19] Y. Chen, E. Gunawan, K. S. Low, S.-C. Wang, C. B. Soh, and L. L. Thi, "Time of arrival data fusion method for two-dimensional ultrawideband breast cancer detection," IEEE Transactions on Antennas and Propagation, vol. 55, no. 10, pp. 2852-2865, 2007.

[20] W. Wei, X. Jin-Yu, and Z. Zhong-Liang, "A new NLOS error mitigation algorithm in location estimation," IEEE Transactions on Vehicular Technology, vol. 54, no. 6, pp. 2048-2053, 2005. 

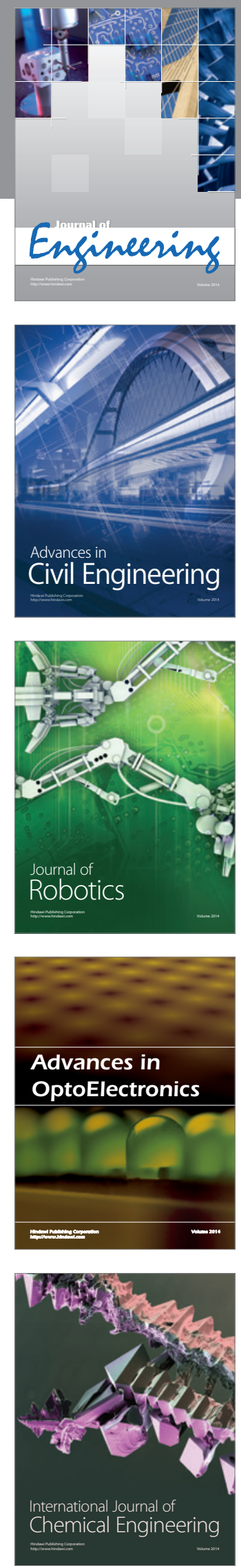

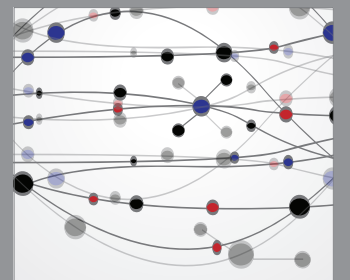

The Scientific World Journal
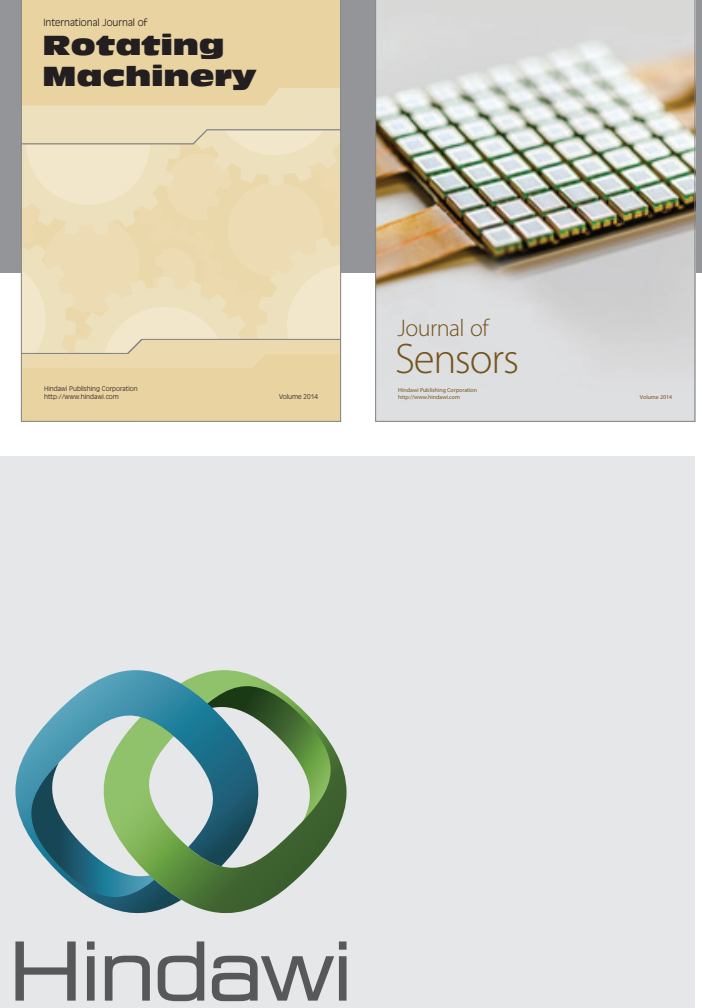

Submit your manuscripts at http://www.hindawi.com
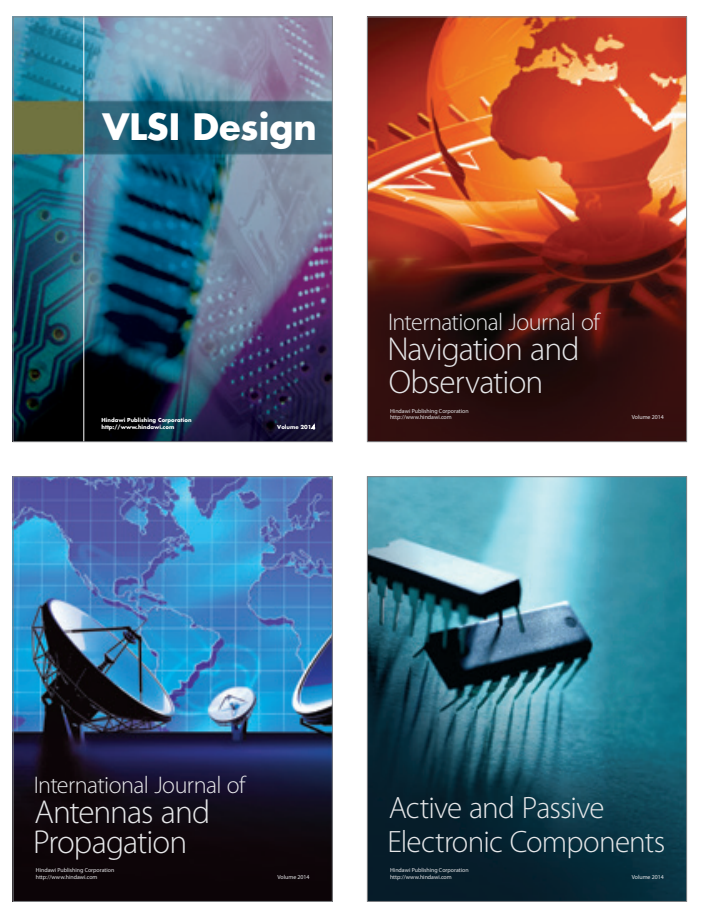
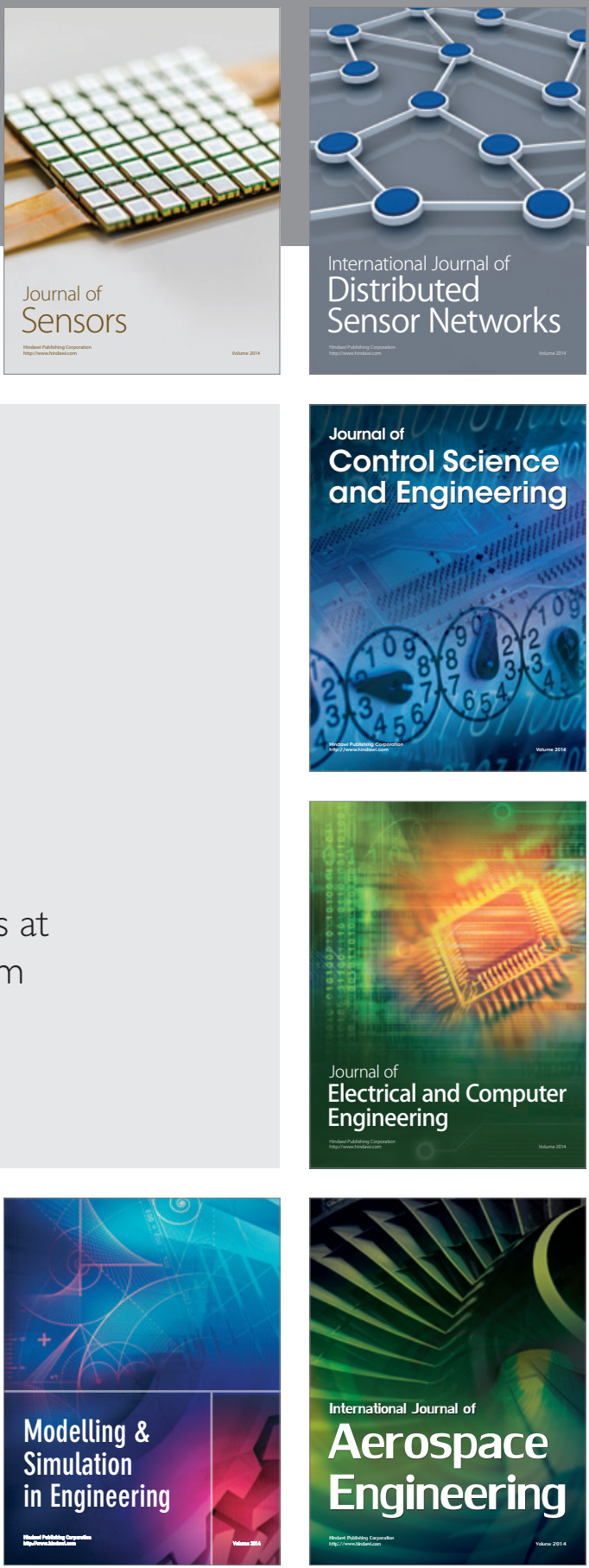

Journal of

Control Science

and Engineering
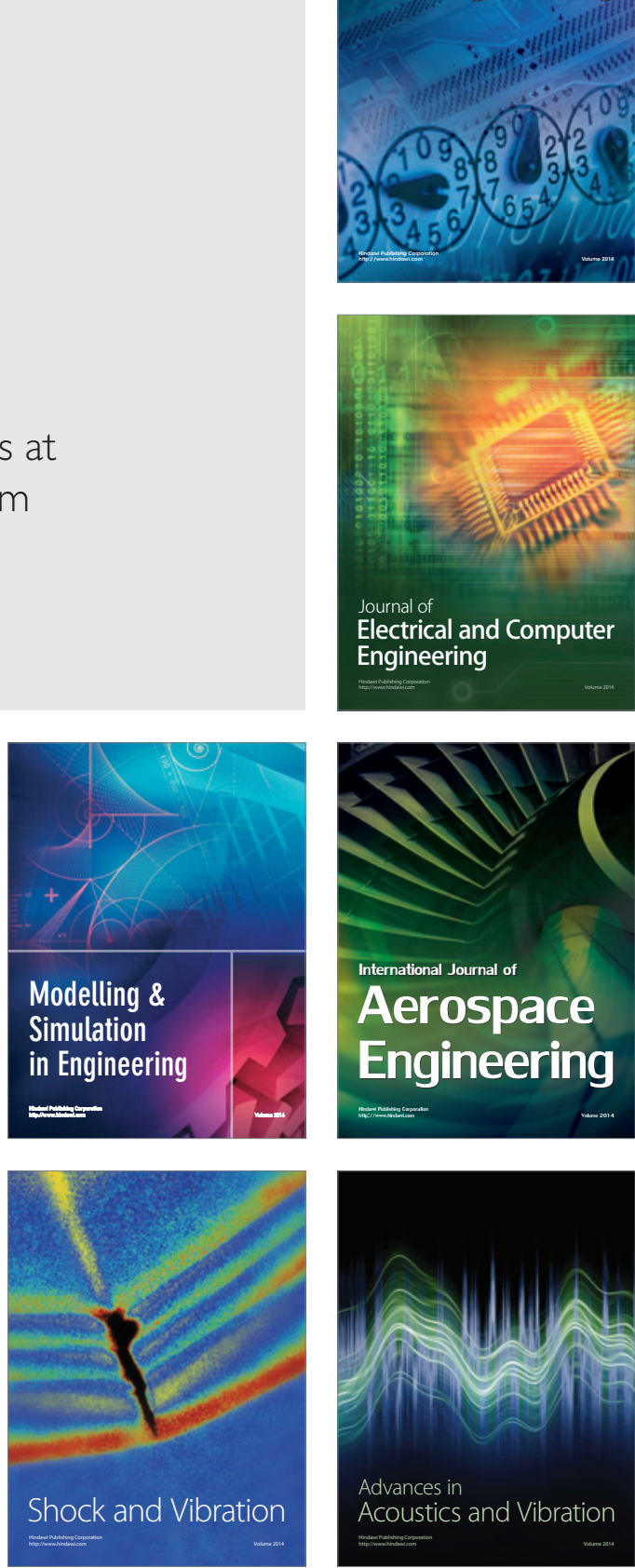